\title{
VectorNet: Encoding HD Maps and Agent Dynamics from Vectorized Representation
}

\author{
Jiyang Gao $^{1 *}$ Chen Sun $^{2 *}$ Hang Zhao $^{1} \quad$ Yi Shen $^{1}$ \\ Dragomir Anguelov ${ }^{1}$ Congcong Li ${ }^{1} \quad$ Cordelia Schmid ${ }^{2}$ \\ ${ }^{1}$ Waymo LLC $\quad{ }^{2}$ Google Research \\ \{jiyanggao, hangz, yshen, dragomir, congcongli\}@waymo.com, \{chensun, cordelias\}@google.com
}

口

\section{Abstract}

Behavior prediction in dynamic, multi-agent systems is an important problem in the context of self-driving cars, due to the complex representations and interactions of road components, including moving agents (e.g. pedestrians and vehicles) and road context information (e.g. lanes, traffic lights). This paper introduces VectorNet, a hierarchical graph neural network that first exploits the spatial locality of individual road components represented by vectors and then models the high-order interactions among all components. In contrast to most recent approaches, which render trajectories of moving agents and road context information as bird-eye images and encode them with convolutional neural networks (ConvNets), our approach operates on a vector representation. By operating on the vectorized high definition (HD) maps and agent trajectories, we avoid lossy rendering and computationally intensive ConvNet encoding steps. To further boost VectorNet's capability in learning context features, we propose a novel auxiliary task to recover the randomly masked out map entities and agent trajectories based on their context. We evaluate VectorNet on our in-house behavior prediction benchmark and the recently released Argoverse forecasting dataset. Our method achieves on par or better performance than the competitive rendering approach on both benchmarks while saving over $70 \%$ of the model parameters with an order of magnitude reduction in FLOPs. It also outperforms the state of the art on the Argoverse dataset.

\section{Introduction}

This paper focuses on behavior prediction in complex multi-agent systems, such as self-driving vehicles. The core interest is to find a unified representation which integrates the agent dynamics, acquired by perception systems such as

\footnotetext{
* equal contribution
}

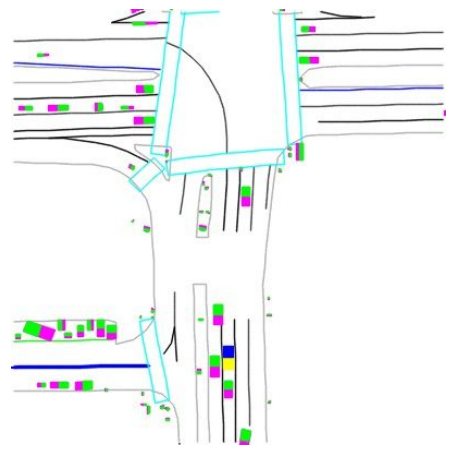

Rasterized Representation

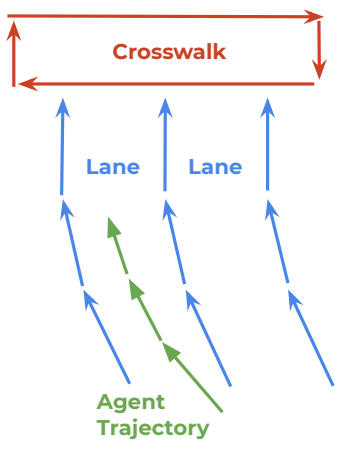

Vectorized Representation
Figure 1. Illustration of the rasterized rendering (left) and vectorized approach (right) to represent high-definition map and agent trajectories.

object detection and tracking, with the scene context, provided as prior knowledge often in the form of High Definition (HD) maps. Our goal is to build a system which learns to predict the intent of vehicles, which are parameterized as trajectories.

Traditional methods for behavior prediction are rulebased, where multiple behavior hypotheses are generated based on constraints from the road maps. More recently, many learning-based approaches are proposed [5, 6, 10, 15]; they offer the benefit of having probabilistic interpretations of different behavior hypotheses, but require building a representation to encode the map and trajectory information. Interestingly, while the HD maps are highly structured, organized as entities with location (e.g. lanes) and attributes (e.g. a green traffic light), most of these approaches choose to render the HD maps as color-coded attributes (Figure 1 , left), which requires manual specifications; and encode the scene context information with ConvNets, which have limited receptive fields. This raise the question: can we learn a meaningful context representation directly from the structured HD maps?

We propose to learn a unified representation for multi- 
Input vectors

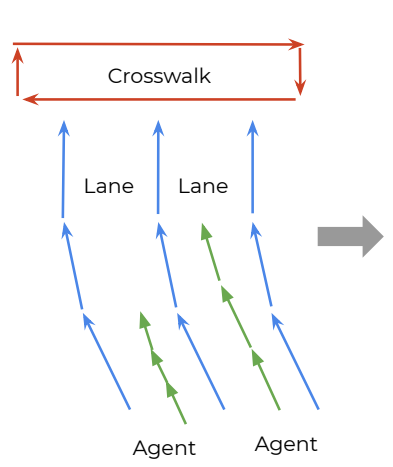

Polyline subgraphs

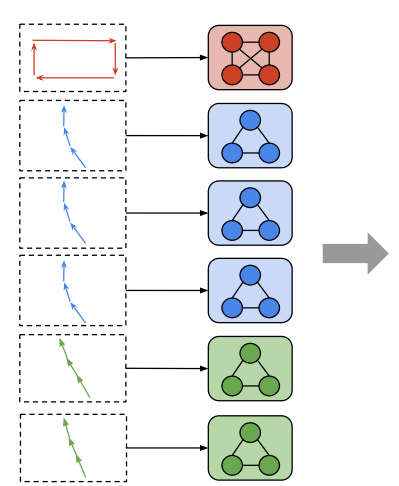

Global interaction graph

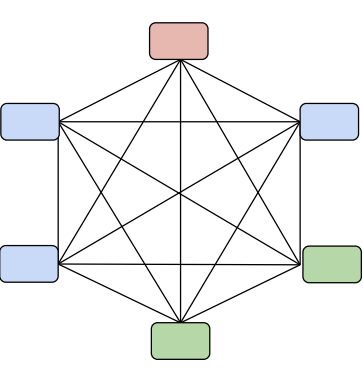

Supervision \& Prediction
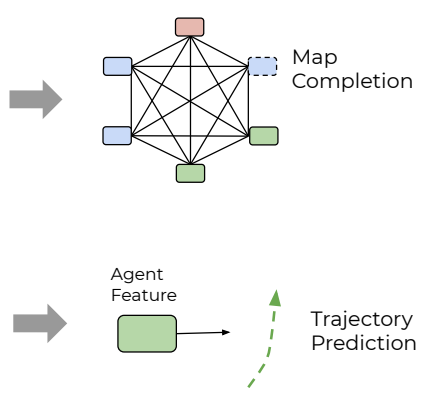

Figure 2. An overview of our proposed VectorNet. Observed agent trajectories and map features are represented as sequence of vectors, and passed to a local graph network to obtain polyline-level features. Such features are then passed to a fully-connected graph to model the higher-order interactions. We compute two types of losses: predicting future trajectories from the node features corresponding to the moving agents and predicting the node features when their features are masked out.

agent dynamics and structured scene context directly from their vectorized form (Figure 1, right). The geographic extent of the road features can be a point, a polygon, or a curve in geographic coordinates. For example, a lane boundary contains multiple control points that build a spline; a crosswalk is a polygon defined by several points; a stop sign is represented by a single point. All these geographic entities can be closely approximated as polylines defined by multiple control points, along with their attributes. Similarly, the dynamics of moving agents can also be approximated by polylines based on their motion trajectories. All these polylines can then be represented as sets of vectors.

We use graph neural networks (GNNs) to incorporate these sets of vectors. We treat each vector as a node in the graph, and set the node features to be the start location and end location of each vector, along with other attributes such as polyline group id and semantic labels. The context information from HD maps, along with the trajectories of other moving agents are propagated to the target agent node through the GNN. We can then take the output node feature corresponding to the target agent to decode its future trajectories.

Specifically, to learn competitive representations with GNNs, we observe that it is important to constrain the connectivities of the graph based on the spatial and semantic proximity of the nodes. We therefore propose a hierarchical graph architecture, where the vectors belonging to the same polylines with the same semantic labels are connected and embedded into polyline features, and all polylines are then fully connected with each other to exchange information. We implement the local graphs with multi-layer perceptrons, and the global graphs with self-attention [30]. An overview of our approach is shown in Figure 2 .

Finally, motivated by the recent success of self- supervised learning from sequential linguistic [11] and visual data [27], we propose an auxiliary graph completion objective in addition to the behavior prediction objective. More specifically, we randomly mask out the input node features belonging to either scene context or agent trajectories, and ask the model to reconstruct the masked features. The intuition is to encourage the graph networks to better capture the interactions between agent dynamics and scene context. In summary, our contributions are:

- We are the first to demonstrate how to directly incorporate vectorized scene context and agent dynamics information for behavior prediction.

- We propose the hierarchical graph network VectorNet and the node completion auxiliary task.

- We evaluate the proposed method on our in-house behavior prediction dataset and the Argoverse dataset, and show that our method achieves on par or better performance over a competitive rendering baseline with $70 \%$ model size saving and an order of magnitude reduction in FLOPs. Our method also achieves the stateof-the-art performance on Argoverse.

\section{Related work}

Behavior prediction for autonomous driving. Behavior prediction for moving agents has become increasingly important for autonomous driving applications [7, 9, 19], and high-fidelity maps have been widely used to provide context information. For example, IntentNet [5] proposes to jointly detect vehicles and predict their trajectories from LiDAR points and rendered HD maps. Hong et al. [15] assumes that vehicle detections are provided and focuses on behavior prediction by encoding entity interactions with ConvNets. Similarly, MultiPath [6] also uses ConvNets as encoder, 
but adopts pre-defined trajectory anchors to regress multiple possible future trajectories. PRECOG [23] attempts to capture the future stochasiticity by flow-based generative models. Similar to [6, 15, 23], we also assume the agent detections to be provided by an existing perception algorithm. However, unlike these methods which all use ConvNets to encode rendered road maps, we propose to directly encode vectorized scene context and agent dynamics.

Forecasting multi-agent interactions. Beyond the autonomous driving domain, there is more general interest to predict the intents of interacting agents, such as for pedestrians [2, 13, 24], human activities [28] or for sports players [12, 26, 32, 33]. In particular, Social LSTM [2] models the trajectories of individual agents as separate LSTM networks, and aggregates the LSTM hidden states based on spatial proximity of the agents to model their interactions. Social GAN [13] simplifies the interaction module and proposes an adversarial discriminator to predict diverse futures. Sun et al. [26] combines graph networks [4] with variational RNNs [8] to model diverse interactions. The social interactions can also be inferred from data: Kipf et al. [18] treats such interactions as latent variables; and graph attention networks [16, 31] apply self-attention mechanism to weight the edges in a pre-defined graph. Our method goes one step further by proposing a unified hierarchical graph network to jointly model the interactions of multiple agents, and their interactions with the entities from road maps.

Representation learning for sets of entities. Traditionally machine perception algorithms have been focusing on highdimensional continuous signals, such as images, videos or audios. One exception is 3D perception, where the inputs are usually in the form of unordered point sets, given by depth sensors. For example, Qi et al. propose the PointNet model [20] and PointNet++ [21] to apply permutation invariant operations (e.g. max pooling) on learned point embeddings. Unlike point sets, entities on HD maps and agent trajectories form closed shapes or are directed, and they may also be associated with attribute information. We therefore propose to keep such information by vectorizing the inputs, and encode the attributes as node features in a graph.

Self-supervised context modeling. Recently, many works in the NLP domain have proposed modeling language context in a self-supervised fashion [11,22]. Their learned representations achieve significant performance improvement when transferred to downstream tasks. Inspired by these methods, we propose an auxiliary loss for graph representations, which learns to predict the missing node features from its neighbors. The goal is to incentivize the model to better capture interactions among nodes.

\section{VectorNet approach}

This section introduces our VectorNet approach. We first describe how to vectorize agent trajectories and HD maps.
Next we present the hierarchical graph network which aggregates local information from individual polylines and then globally over all trajectories and map features. This graph can then be used for behavior prediction.

\subsection{Representing trajectories and maps}

Most of the annotations from an HD map are in the form of splines (e.g. lanes), closed shape (e.g. regions of intersections) and points (e.g. traffic lights), with additional attribute information such as the semantic labels of the annotations and their current states (e.g. color of the traffic light, speed limit of the road). For agents, their trajectories are in the form of directed splines with respect to time. All of these elements can be approximated as sequences of vectors: for map features, we pick a starting point and direction, uniformly sample key points from the splines at the same spatial distance, and sequentially connect the neighboring key points into vectors; for trajectories, we can just sample key points with a fixed temporal interval $(0.1 \mathrm{sec}-$ ond), starting from $t=0$, and connect them into vectors. Given small enough spatial or temporal intervals, the resulting polylines serve as close approximations of the original map and trajectories.

Our vectorization process is a one-to-one mapping between continuous trajectories, map annotations and the vector set, although the latter is unordered. This allows us to form a graph representation on top of the vector sets, which can be encoded by graph neural networks. More specifically, we treat each vector $\mathbf{v}_{i}$ belonging to a polyline $\mathcal{P}_{j}$ as a node in the graph with node features given by

$$
\mathbf{v}_{i}=\left[\mathbf{d}_{i}^{s}, \mathbf{d}_{i}^{e}, \mathbf{a}_{i}, j\right]
$$

where $\mathbf{d}_{i}^{s}$ and $\mathbf{d}_{i}^{e}$ are coordinates of the start and end points of the vector, $\mathbf{d}$ itself can be represented as $(x, y)$ for $2 \mathrm{D}$ coordinates or $(x, y, z)$ for $3 \mathrm{D}$ coordinates; $\mathbf{a}_{i}$ corresponds to attribute features, such as object type, timestamps for trajectories, or road feature type or speed limit for lanes; $j$ is the integer id of $\mathcal{P}_{j}$, indicating $\mathbf{v}_{i} \in \mathcal{P}_{j}$.

To make the input node features invariant to the locations of target agents, we normalize the coordinates of all vectors to be centered around the location of target agent at its last observed time step. A future work is to share the coordinate centers for all interacting agents, such that their trajectories can be predicted in parallel.

\subsection{Constructing the polyline subgraphs}

To exploit the spatial and semantic locality of the nodes, we take a hierarchical approach by first constructing subgraphs at the vector level, where all vector nodes belonging to the same polyline are connected with each other. Considering a polyline $\mathcal{P}$ with its nodes $\left\{\mathbf{v}_{1}, \mathbf{v}_{2}, \ldots, \mathbf{v}_{P}\right\}$, we define a single layer of subgraph propagation operation as

$$
\mathbf{v}_{i}^{(l+1)}=\varphi_{\text {rel }}\left(g_{\text {enc }}\left(\mathbf{v}_{i}^{(l)}\right), \varphi_{\text {agg }}\left(\left\{g_{\text {enc }}\left(\mathbf{v}_{j}^{(l)}\right)\right\}\right)\right)
$$




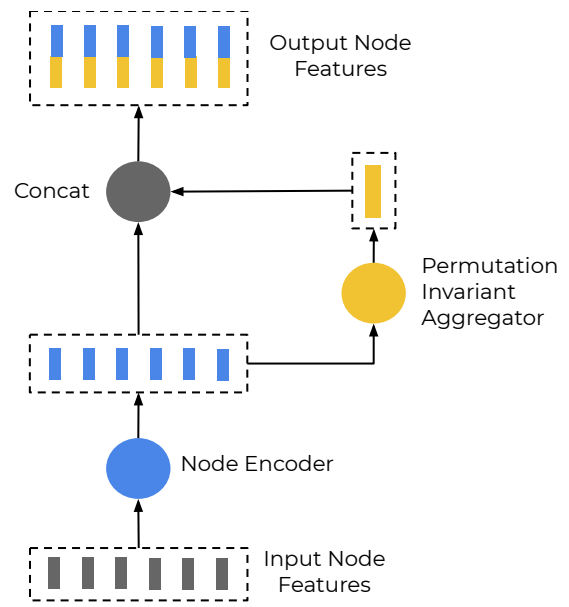

Figure 3. The computation flow on the vector nodes of the same polyline.

where $\mathbf{v}_{i}^{(l)}$ is the node feature for $l$-th layer of the subgraph network, and $\mathbf{v}_{i}^{(0)}$ is the input features $\mathbf{v}_{i}$. Function $g_{\text {enc }}(\cdot)$ transforms the individual node features, $\varphi_{\mathrm{agg}}(\cdot)$ aggregates the information from all neighboring nodes, and $\varphi_{\text {rel }}(\cdot)$ is the relational operator between node $\mathbf{v}_{i}$ and its neighbors.

In practice, $g_{\mathrm{enc}}(\cdot)$ is a multi-layer perceptron (MLP) whose weights are shared over all nodes; specifically, the MLP contains a single fully connected layer followed by layer normalization [3] and then ReLU non-linearity. $\varphi_{\text {agg }}(\cdot)$ is the maxpooling operation, and $\varphi_{\text {rel }}(\cdot)$ is a simple concatenation. An illustration is shown in Figure 3. We stack multiple layers of the subgraph networks, where the weights for $g_{\text {enc }}(\cdot)$ are different. Finally, to obtain polyline level features, we compute

$$
\mathbf{p}=\varphi_{\mathrm{agg}}\left(\left\{\mathbf{v}_{i}^{\left(L_{p}\right)}\right\}\right)
$$

where $\varphi_{\text {agg }}(\cdot)$ is again maxpooling.

Our polyline subgraph network can be seen as a generalization of PointNet [20]: when we set $\mathbf{d}^{s}=\mathbf{d}^{e}$ and let $\mathbf{a}$ and $l$ to be empty, our network has the same inputs and compute flow as PointNet. However, by embedding the ordering information into vectors, constraining the connectivity of subgraphs based on the polyline groupings, and encoding attributes as node features, our method is particularly suitable to encode structured map annotations and agent trajectories.

\subsection{Global graph for high-order interactions}

We now consider modeling the high-order interactions on the polyline node features $\left\{\mathbf{p}_{1}, \mathbf{p}_{2}, \ldots, \mathbf{p}_{P}\right\}$ with a global interaction graph:

$$
\left\{\mathbf{p}_{i}^{(l+1)}\right\}=\operatorname{GNN}\left(\left\{\mathbf{p}_{i}^{(l)}\right\}, \mathcal{A}\right)
$$

where $\left\{\mathbf{p}_{i}^{(l)}\right\}$ is the set of polyline node features, $\operatorname{GNN}(\cdot)$ corresponds to a single layer of a graph neural network, and $\mathcal{A}$ corresponds to the adjacency matrix for the set of polyline nodes.

The adjacency matrix $\mathcal{A}$ can be provided a heuristic, such as using the spatial distances [2] between the nodes. For simplicity, we assume $\mathcal{A}$ to be a fully-connected graph. Our graph network is implemented as a self-attention operation [30]:

$$
\operatorname{GNN}(\mathbf{P})=\operatorname{softmax}\left(\mathbf{P}_{Q} \mathbf{P}_{K}^{T}\right) \mathbf{P}_{V}
$$

where $\mathbf{P}$ is the node feature matrix and $\mathbf{P}_{Q}, \mathbf{P}_{K}$ and $\mathbf{P}_{V}$ are its linear projections.

We then decode the future trajectories from the nodes corresponding the moving agents:

$$
\mathbf{v}_{i}^{\text {future }}=\varphi_{\text {traj }}\left(\mathbf{p}_{i}^{\left(L_{t}\right)}\right)
$$

where $L_{t}$ is the number of the total number of GNN layers, and $\varphi_{\text {traj }}(\cdot)$ is the trajectory decoder. For simplicity, we use an MLP as the decoder function. More advanced decoders, such as the anchor-based approach from MultiPath [6], or variational RNNs [8, 26] can be used to generate diverse trajectories; these decoders are complementary to our input encoder.

We use a single GNN layer in our implementation, so that during inference time, only the node features corresponding to the target agents need to be computed. However, we can also stack multiple layers of $\mathrm{GNN}(\cdot)$ to model higher-order interactions when needed.

To encourage our global interaction graph to better capture interactions among different trajectories and map polylines, we introduce an auxiliary graph completion task. During training time, we randomly mask out the features for a subset of polyline nodes, e.g. $\mathbf{p}_{i}$. We then attempt to recover its masked out feature as:

$$
\hat{\mathbf{p}}_{i}=\varphi_{\text {node }}\left(\mathbf{p}_{i}^{\left(L_{t}\right)}\right)
$$

where $\varphi_{\text {node }}(\cdot)$ is the node feature decoder implemented as an MLP. These node feature decoders are not used during inference time.

Recall that $\mathbf{p}_{i}$ is a node from a fully-connected, unordered graph. In order to identify an individual polyline node when its corresponding feature is masked out, we compute the minimum values of the start coordinates from all of its belonging vectors to obtain the identifier embedding $\mathbf{p}_{i}^{\text {id }}$. The inputs node features then become

$$
\mathbf{p}_{i}^{(0)}=\left[\mathbf{p}_{i} ; \mathbf{p}_{i}^{\text {id }}\right]
$$

Our graph completion objective is closely related to the widely successful BERT [11] method for natural language 
processing, which predicts missing tokens based on bidirectional context from discrete and sequential text data. We generalize this training objective to work with unordered graphs. Unlike several recent methods (e.g. [25]) that generalizes the BERT objective to unordered image patches with pre-computed visual features, our node features are jointly optimized in an end-to-end framework.

\subsection{Overall framework}

Once the hierarchical graph network is constructed, we optimize for the multi-task training objective

$$
\mathcal{L}=\mathcal{L}_{\text {traj }}+\alpha \mathcal{L}_{\text {node }}
$$

where $\mathcal{L}_{\text {traj }}$ is the negative Gaussian log-likelihood for the groundtruth future trajectories, $\mathcal{L}_{\text {node }}$ is the Huber loss between predicted node features and groundtruth masked node features, and $\alpha=1.0$ is a scalar that balances the two loss terms. To avoid trivial solutions for $\mathcal{L}_{\text {node }}$ by lowering the magnitude of node features, we L2 normalize the polyline node features before feeding them to the global graph network.

Our predicted trajectories are parameterized as per-step coordinate offsets, starting from the last observed location. We rotate the coordinate system based on the heading of the target vehicle at the last observed location.

\section{Experiments}

In this section, we first describe the experimental settings, including the datasets, metrics and rasterized + ConvNets baseline. Secondly, comprehensive ablation studies are done for both the rasterized baseline and VectorNet. Thirdly, we compare and discuss the computation cost, including FLOPs and number of parameters. Finally, we compare the performance with state-of-the-art methods.

\subsection{Experimental setup}

\subsubsection{Datasets}

We report results on two vehicle behavior prediction benchmarks, the recently released Argoverse dataset [7] and our in-house behavior prediction dataset.

Argoverse motion forecasting [7] is a dataset designed for vehicle behavior prediction with trajectory histories. There are $333 \mathrm{~K} 5$-second long sequences split into $211 \mathrm{~K}$ training, $41 \mathrm{~K}$ validation and $80 \mathrm{~K}$ testing sequences. The creators curated this dataset by mining interesting and diverse scenarios, such as yielding for a merging vehicle, crossing an intersection, etc. The trajectories are sampled at $10 \mathrm{~Hz}$, with $(0,2]$ seconds are used as observation and $(2,5]$ seconds for trajectory prediction. Each sequence has one "interesting" agent whose trajectory is the prediction target. In addition to vehicle trajectories, each sequence is also associated with map information. The future trajectories of the test set are held out. Unless otherwise mentioned, our ablation study reports performance on the validation set.

In-house dataset is a large-scale dataset collected for behavior prediction. It contains HD map data, bounding boxes and tracks obtained with an automatic in-house perception system, and manually labeled vehicle trajectories. The total number of vehicle trajectories are $2.2 \mathrm{M}$ and $0.55 \mathrm{M}$ for train and test sets. Each trajectory has a length of 4 seconds, where the $(0,1]$ second is the history trajectory used as observation, and $(1,4]$ seconds are the target future trajectories to be evaluated. The trajectories are sampled from real world vehicles' behaviors, including stationary, going straight, turning, lane change and reversing, and roughly preserves the natural distribution of driving scenarios. For the HD map features, we include lane boundaries, stop/yield signs, crosswalks and speed bumps.

For both datasets, the input history trajectories are derived from automatic perception systems and are thus noisy. Argoverse's future trajectories are also machine generated, while In-house has manually labeled future trajectories.

\subsubsection{Metrics}

For evaluation we adopt the widely used Average Displacement Error (ADE) computed over the entire trajectories and the Displacement Error at $t$ (DE@ts) metric, where $t \in\{1.0,2.0,3.0\}$ seconds. The displacements are measured in meters.

\subsubsection{Baseline with rasterized images}

We render $N$ consecutive past frames, where $N$ is 10 for the in-house dataset and 20 for the Argoverse dataset. Each frame is a $400 \times 400 \times 3$ image, which has road map information and the detected object bounding boxes. 400 pixels correspond to 100 meters in the in-house dataset, and 130 meters in the Argoverse dataset. Rendering is based on the position of self-driving vehicle in the last observed frame; the self-driving vehicle is placed at the coordinate location $(200,320)$ in in-house dataset, and $(200,200)$ in Argoverse dataset. All $N$ frames are stacked together to form a $400 \times 400 \times 3 \mathrm{~N}$ image as model input.

Our baseline uses a ConvNet to encode the rasterized images, whose architecture is comparable to IntentNet [5]: we use a ResNet-18 [14] as the ConvNet backbone. Unlike IntentNet, we do not use the LiDAR inputs. To obtain vehicle-centric features, we crop the feature patch around the target vehicle from the convolutional feature map, and average pool over all the spatial locations of the cropped feature map to get a single vehicle feature vector. We empirically observe that using a deeper ResNet model or rotating the cropped features based on target vehicle headings do not lead to better performance. The vehicle features are 
then fed into a fully connected layer (as used by IntentNet) to predict the future coordinates in parallel. The model is optimized on 8 GPUs with synchronous training. We use the Adam optimizer [17] and decay the learning rate every 5 epochs by a factor of 0.3 . We train the model for a total of 25 epochs with an initial learning rate of 0.001 .

To test how convolutional receptive fields and feature cropping strategies influence the performance, we conduct ablation study on the network receptive field, feature cropping strategy and input image resolutions.

\subsubsection{VectorNet with vectorized representations}

To ensure a fair comparison, the vectorized representation takes as input the same information as the rasterized representation. Specifically, we extract exactly the same set of map features as when rendering. We also make sure that the visible road feature vectors for a target agent are the same as in the rasterized representation. However, the vectorized representation does enjoy the benefit of incorporating more complex road features which are non-trivial to render.

Unless otherwise mentioned, we use three graph layers for the polyline subgraphs, and one graph layer for the global interaction graph. The number of hidden units in all MLPs are fixed to 64. The MLPs are followed by layer normalization and ReLU nonlinearity. We normalize the vector coordinates to be centered around the location of target vehicle at the last observed time step. Similar to the rasterized model, VectorNet is trained on 8 GPUs synchronously with Adam optimizer. The learning rate is decayed every 5 epochs by a factor of 0.3 , we train the model for a total of 25 epochs with initial learning rate of 0.001 .

To understand the impact of the components on the performance of VectorNet, we conduct ablation studies on the type of context information, i.e. whether to use only map or also the trajectories of other agents as well as the impact of number of graph layers for the polyline subgraphs and global interaction graphs.

\subsection{Ablation study for the ConvNet baseline}

We conduct ablation studies on the impact of ConvNet receptive fields, feature cropping strategies, and the resolution of the rasterized images.

Impact of receptive fields. As behavior prediction often requires capturing long range road context, the convolutional receptive field could be critical to the prediction quality. We evaluate different variants to see how two key factors of receptive fields, convolutional kernel sizes and feature cropping strategies, affect the prediction performance. The results are shown in Table 1. By comparing kernel size 3, 5 and 7 at $400 \times 400$ resolution, we can see that a larger kernel size leads to slight performance improvement. However, it also leads to quadratic increase of the computation cost. We also compare different cropping methods, by increasing the crop size or cropping along the vehicle trajectory at all observed time steps. From the 3 rd to 6 th rows of Table 1 we can see that a larger crop size (3 v.s. 1) can significantly improve the performance, and cropping along observed trajectory also leads to better performance. This observation confirms the importance of receptive fields when rasterized images are used as inputs. It also highlights its limitation, where a carefully designed cropping strategy is needed, often at the cost of increased computation cost.

Impact of rendering resolution. We further vary the resolutions of rasterized images to see how it affects the prediction quality and computation cost, as shown in the first three rows of Table 1 . We test three different resolutions, including $400 \times 400(0.25$ meter per pixel $), 200 \times 200(0.5$ meter per pixel) and $100 \times 100(1$ meter per pixel). It can be seen that the performance increases generally as the resolution goes up. However, for the Argoverse dataset we can see that increasing the resolution from $200 \times 200$ to $400 \times 400$ leads to slight drop in performance, which can be explained by the decrease of effective receptive field size with the fixed $3 \times 3$ kernel. We discuss the impact on computation cost of these design choices in Section 4.4

\subsection{Ablation study for VectorNet}

Impact of input node types. We study whether it is helpful to incorporate both map features and agent trajectories for VectorNet. The first three rows in Table 2 correspond to using only the past trajectory of the target vehicle ("none" context), adding only map polylines ("map"), and finally adding trajectory polylines ("map + agents"). We can clearly observe that adding map information significantly improves the trajectory prediction performance. Incorporating trajectory information furthers improves the performance.

Impact of node completion loss. The last four rows of Table 2 compares the impact of adding the node completion auxiliary objective. We can see that adding this objective consistently helps with performance, especially at longer time horizons.

Impact on the graph architectures. In Table 3 we study the impact of depths and widths of the graph layers on trajectory prediction performance. We observe that for the polyline subgraph three layers gives the best performance, and for the global graph just one layer is needed. Making the MLPs wider does not lead to better performance, and hurts for Argoverse, presumably because it has a smaller training dataset. Some example visualizations on predicted trajectory and lane attention are shown in Figure 4.

Comparison with ConvNets. Finally, we compare our VectorNet with the best ConvNet model in Table 4. For the in-house dataset, our model achieves on par performance with the best ResNet model, while being much more eco- 


\begin{tabular}{|c|c|c||c|c|c|c|c|c|c|c|}
\hline Resolution & \multicolumn{1}{|c||}{ Kernel } & \multicolumn{1}{c|}{ Crop } & \multicolumn{4}{|c|}{ In-house dataset } & \multicolumn{4}{c|}{ Argoverse dataset } \\
\cline { 4 - 11 } & & & DE@ 1s & DE@2s & DE@3s & ADE & DE@ 1s & DE@ 2s & DE@ 3s & ADE \\
\hline \hline $100 \times 100$ & $3 \times 3$ & $1 \times 1$ & 0.63 & 0.94 & 1.32 & 0.82 & 1.14 & 2.80 & 5.19 & 2.21 \\
$200 \times 200$ & $3 \times 3$ & $1 \times 1$ & 0.57 & 0.86 & 1.21 & 0.75 & 1.11 & 2.72 & 4.96 & 2.15 \\
\hline $400 \times 400$ & $3 \times 3$ & $1 \times 1$ & 0.55 & 0.82 & 1.16 & 0.72 & 1.12 & 2.72 & 4.94 & 2.16 \\
$400 \times 400$ & $3 \times 3$ & $3 \times 3$ & 0.50 & 0.77 & 1.09 & 0.68 & 1.09 & 2.62 & 4.81 & 2.08 \\
$400 \times 400$ & $3 \times 3$ & $5 \times 5$ & 0.50 & 0.76 & 1.08 & 0.67 & 1.09 & 2.60 & 4.70 & 2.08 \\
$400 \times 400$ & $3 \times 3$ & traj & 0.47 & 0.71 & 1.00 & 0.63 & 1.05 & 2.48 & 4.49 & 1.96 \\
\hline $400 \times 400$ & $5 \times 5$ & $1 \times 1$ & 0.54 & 0.81 & 1.16 & 0.72 & 1.10 & 2.63 & 4.75 & 2.13 \\
$400 \times 400$ & $7 \times 7$ & $1 \times 1$ & 0.53 & 0.81 & 1.16 & 0.72 & 1.10 & 2.63 & 4.74 & 2.13 \\
\hline
\end{tabular}

Table 1. Impact of receptive field (as controlled by convolutional kernel size and crop strategy) and rendering resolution for the ConvNet baseline. We report DE and ADE (in meters) on both the in-house dataset and the Argoverse dataset.

\begin{tabular}{|c|c||c|c|c|c|c|c|c|c|}
\hline \multirow{2}{*}{ Context } & \multicolumn{1}{|c|}{ Node Compl. } & \multicolumn{4}{|c|}{ In-house dataset } & \multicolumn{4}{c|}{ Argoverse dataset } \\
\cline { 3 - 10 } & & DE@ 1s & DE@ 2s & DE@ 3s & ADE & DE@ 1s & DE@ 2s & DE@3s & ADE \\
\hline \hline none & - & 0.77 & 0.99 & 1.29 & 0.92 & 1.29 & 2.98 & 5.24 & 2.36 \\
\hline map & no & 0.57 & 0.81 & 1.11 & 0.72 & 0.95 & 2.18 & 3.94 & 1.75 \\
map + agents & no & 0.55 & 0.78 & 1.05 & 0.70 & 0.94 & 2.14 & 3.84 & 1.72 \\
\hline map & yes & 0.55 & 0.78 & 1.07 & 0.70 & 0.94 & 2.11 & 3.77 & 1.70 \\
map + agents & yes & $\mathbf{0 . 5 3}$ & $\mathbf{0 . 7 4}$ & $\mathbf{1 . 0 0}$ & $\mathbf{0 . 6 6}$ & $\mathbf{0 . 9 2}$ & $\mathbf{2 . 0 6}$ & $\mathbf{3 . 6 7}$ & $\mathbf{1 . 6 6}$ \\
\hline
\end{tabular}

Table 2. Ablation studies for VectorNet with different input node types and training objectives. Here "map" refers to the input vectors from the HD maps, and "agents" refers to the input vectors from the trajectories of non-target vehicles. When "Node Compl." is enabled, the model is trained with the graph completion objective in addition to trajectory prediction. DE and ADE are reported in meters.

\begin{tabular}{|c|c|c|c|c|c|}
\hline \multicolumn{2}{|c|}{ Polyline Subgraph } & \multicolumn{2}{|c|}{ Global Graph } & \multicolumn{2}{c|}{ DE@ 3s } \\
\hline Depth & Width & Depth & Width & In-house & Argoverse \\
\hline \hline 1 & 64 & 1 & 64 & 1.09 & 3.89 \\
3 & 64 & 1 & 64 & 1.00 & 3.67 \\
3 & 128 & 1 & 64 & 1.00 & 3.93 \\
3 & 64 & 2 & 64 & 0.99 & 3.69 \\
3 & 64 & 2 & 256 & 1.02 & 3.69 \\
\hline
\end{tabular}

Table 3. Ablation on the depth and width of polyline subgraph and global graph. The depth of polyline subgraph has biggest impact on DE@3s.

nomically in terms of model size and FLOPs. For the Argoverse dataset, our approach significantly outperforms the best ConvNet model with 12\% reduction in DE@3. We observe that the in-house dataset contains a lot of stationary vehicles due to its natural distribution of driving scenarios; those cases can be easily solved by ConvNets, which are good at capturing local pattern. However, for the Argoverse dataset where only "interesting" cases are preserved, VectorNet outperforms the best ConvNet baseline by a large margin; presumably due to its ability to capture long range context information via the hierarchical graph network.

\subsection{Comparison of FLOPs and model size}

We now compare the FLOPs and model size between ConvNets and VectorNet, and their implications on performance. The results are shown in Table 4 The prediction decoder is not counted for FLOPs and number of parameters. We can see that the FLOPs of ConvNets increase quadrati-

\begin{tabular}{|c||c|c|c|c|}
\hline \multicolumn{1}{|c||}{ Model } & FLOPs & \#Param & \multicolumn{2}{|c|}{ DE@ 3s } \\
\cline { 4 - 5 } & & & In-house & Argo \\
\hline \hline R18-k3-c1-r100 & $0.66 \mathrm{G}$ & $246 \mathrm{~K}$ & 1.32 & 5.19 \\
R18-k3-c1-r200 & $2.64 \mathrm{G}$ & $246 \mathrm{~K}$ & 1.21 & 4.95 \\
R18-k3-c1-r400 & $10.56 \mathrm{G}$ & $246 \mathrm{~K}$ & 1.16 & 4.96 \\
R18-k5-c1-r400 & $15.81 \mathrm{G}$ & $509 \mathrm{~K}$ & 1.16 & 4.75 \\
R18-k7-c1-r400 & $23.67 \mathrm{G}$ & $902 \mathrm{~K}$ & 1.16 & 4.74 \\
R18-k3-c3-r400 & $10.56 \mathrm{G}$ & $246 \mathrm{~K}$ & 1.09 & 4.81 \\
R18-k3-c5-r400 & $10.56 \mathrm{G}$ & $246 \mathrm{~K}$ & 1.08 & 4.70 \\
R18-k3-t-r400 & $10.56 \mathrm{G}$ & $246 \mathrm{~K}$ & 1.00 & 4.49 \\
\hline VectorNet w/o aux. & $0.041 \mathrm{G} \times \mathrm{n}$ & $72 \mathrm{~K}$ & 1.05 & 3.84 \\
VectorNet w aux. & $0.041 \mathrm{G} \times \mathrm{n}$ & $72 \mathrm{~K}$ & $\mathbf{1 . 0 0}$ & $\mathbf{3 . 6 7}$ \\
\hline
\end{tabular}

Table 4. Model FLOPs and number of parameters comparison for ResNet and VectorNet. R18-k $M$-c $N$-r $S$ stands for the ResNet-18 model with kernel size $M \times M$, crop patch size $N \times N$ and input resolution $S \times S$. Prediction decoder is not counted for FLOPs and parameters.

cally with the kernel size and input image size; the number of parameters increases quadratically with the kernel size. As we render the images centered at the self driving vehicle, the feature map can be reused among multiple targets, so the FLOPs of the backbone part is a constant number. However, if the rendered images are target-centered, the FLOPs increases linearly with the number of targets. For VectorNet, the FLOPs depends on the number of vector nodes and polylines in the scene. For the in-house dataset, the average number of road map polylines is 17 containing 205 vectors; the average number of road agent polylines is 59 contain- 

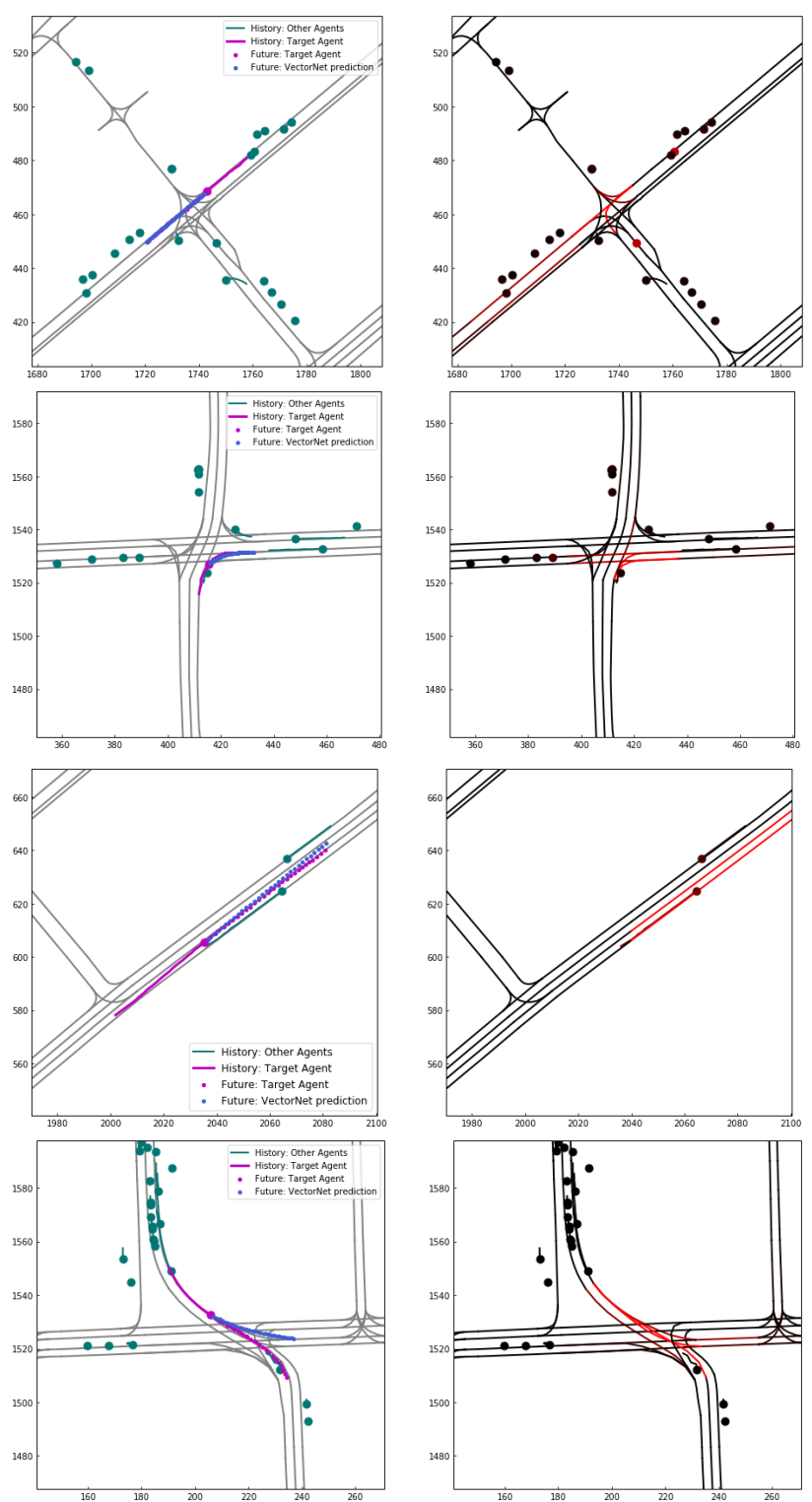

Figure 4. (Left) Visualization of the prediction: lanes are shown in grey, non-target agents are green, target agent's ground truth trajectory is in pink, predicted trajectory in blue. (Right) Visualization of attention for road and agent: Brighter red color corresponds to higher attention score. It can be seen that when agents are facing multiple choices (first two examples), the attention mechanism is able to focus on the correct choices (two right-turn lanes in the second example). The third example is a lane-changing agent, the attended lanes are the current lane and target lane. In the fourth example, though the prediction is not accurate, the attention still produces a reasonable score on the correct lane.

ing 590 vectors. We calculate the FLOPs based on these average numbers. Note that, as we need to re-normalize the vector coordinates and re-compute the VectorNet features for each target, the FLOPs increase linearly with the number of predicting targets ( $n$ in Table 4 ).

\begin{tabular}{c||cc}
\hline Model & DE@3s & ADE \\
\hline \hline Constant Velocity [7] & 7.89 & 3.53 \\
Nearest Neighbor [7] & 7.88 & 3.45 \\
LSTM ED [7] & 4.95 & 2.15 \\
Challenge Winner: uulm-mrm & 4.19 & 1.90 \\
Challenge Winner: Jean & 4.17 & 1.86 \\
\hline VectorNet & $\mathbf{4 . 0 1}$ & $\mathbf{1 . 8 1}$ \\
\hline
\end{tabular}

Table 5. Trajectory prediction performance on the Argoverse Forecasting test set when number of sampled trajectories $\mathrm{K}=1$. Results were retrieved from the Argoverse leaderboard [1] on 03/18/2020.

Comparing R18-k3-t-r400 (the best model among ConvNets) with VectorNet, VectorNet significantly outperforms ConvNets. For computation, ConvNets consumes 200+ times more FLOPs than VectorNet $(10.56 \mathrm{G}$ vs $0.041 \mathrm{G})$ for a single agent; considering that the average number of vehicles in a scene is around 30 (counted from the in-house dataset), the actual computation consumption of VectorNet is still much smaller than that of ConvNets. At the same time, VectorNet needs $29 \%$ of the parameters of ConvNets ( $72 \mathrm{~K}$ vs $246 \mathrm{~K})$. Based on the comparison, we can see that VectorNet can significantly boost the performance while at the same time dramatically reducing computation cost.

\subsection{Comparison with state-of-the-art methods}

Finally, we compare VectorNet with several baseline approaches [7] and some state-of-the-art methods on the Argoverse [7] test set. We report $\mathrm{K}=1$ results (the most likely predictions) in Table 5. The baseline approaches include the constant velocity baseline, nearest neighbor retrieval, and LSTM encoder-decoder. The state-of-the-art approaches are the winners of Argoverse Forecasting Challenge. It can be seen that VectorNet improves the state-of-the-art performance from 4.17 to 4.01 for the $\mathrm{DE} @ 3 \mathrm{~s}$ metric when $\mathrm{K}=1$.

\section{Conclusion and future work}

We proposed to represent the HD map and agent dynamics with a vectorized representation. We designed a novel hierarchical graph network, where the first level aggregates information among vectors inside a polyline, and the second level models the higher-order relationships among polylines. Experiments on the large scale in-house dataset and the public available Argoverse dataset show that the proposed VectorNet outperforms the ConvNet counterpart while at the same time reducing the computational cost by a large margin. VectorNet also achieves state-of-the-art performance (DE@3s, K=1) on the Argoverse test set. A natural next step is to incorporate the VectorNet encoder with a multi-modal trajectory decoder (e.g. [6, 29]) to generate diverse future trajectories.

Acknowledgement. We want to thank Benjamin Sapp and Yuning Chai for their helpful comments on the paper. 


\section{References}

[1] Argoverse Motion Forecasting Competition, 2019. https://evalai.cloudcv.org/web/challenges/ challenge-page/454/leaderboard/1279

[2] Alexandre Alahi, Kratarth Goel, Vignesh Ramanathan, Alexandre Robicquet, Li Fei-Fei, and Silvio Savarese. Social LSTM: Human Trajectory Prediction in Crowded Spaces. In CVPR, 2016.

[3] Jimmy Lei Ba, Jamie Ryan Kiros, and Geoffrey E Hinton. Layer normalization. arXiv preprint arXiv:1607.06450, 2016.

[4] Peter W Battaglia, Jessica B Hamrick, Victor Bapst, Alvaro Sanchez-Gonzalez, Vinicius Zambaldi, Mateusz Malinowski, Andrea Tacchetti, David Raposo, Adam Santoro, Ryan Faulkner, Caglar Gulcehre, Francis Song, Andrew Ballard, Justin Gilmer, George Dahl, Ashish Vaswani, Kelsey Allen, Charles Nash, Victoria Langston, Chris Dyer, Nicolas Heess, Daan Wierstra, Pushmeet Kohli, Matt Botvinick, Oriol Vinyals, Yujia Li, and Razvan Pascanu. Relational inductive biases, deep learning, and graph networks. arXiv preprint arXiv:1806.01261, 2018.

[5] Sergio Casas, Wenjie Luo, and Raquel Urtasun. Intentnet: Learning to predict intention from raw sensor data. In CoRL, 2018.

[6] Yuning Chai, Benjamin Sapp, Mayank Bansal, and Dragomir Anguelov. Multipath: Multiple probabilistic anchor trajectory hypotheses for behavior prediction. In CoRL, 2019.

[7] Ming-Fang Chang, John Lambert, Patsorn Sangkloy, Jagjeet Singh, Slawomir Bak, Andrew Hartnett, De Wang, Peter Carr, Simon Lucey, Deva Ramanan, et al. Argoverse: 3D tracking and forecasting with rich maps. In $C V P R, 2019$.

[8] Junyoung Chung, Kyle Kastner, Laurent Dinh, Kratarth Goel, Aaron C Courville, and Yoshua Bengio. A recurrent latent variable model for sequential data. In NeurIPS, 2015.

[9] James Colyar and Halkias John. Us highway 101 dataset. FHWAHRT-07-030, 2007.

[10] Henggang Cui, Vladan Radosavljevic, Fang-Chieh Chou, TsungHan Lin, Thi Nguyen, Tzu-Kuo Huang, Jeff Schneider, and Nemanja Djuric. Multimodal trajectory predictions for autonomous driving using deep convolutional networks. In ICRA, 2019.

[11] Jacob Devlin, Ming-Wei Chang, Kenton Lee, and Kristina Toutanova. BERT: Pre-training of deep bidirectional transformers for language understanding. arXiv preprint arXiv:1810.04805, 2018.

[12] Panna Felsen, Pulkit Agrawal, and Jitendra Malik. What will happen next? forecasting player moves in sports videos. In ICCV, 2017.

[13] Agrim Gupta, Justin Johnson, Li Fei-Fei, Silvio Savarese, and Alexandre Alahi. Social GAN: Socially acceptable trajectories with generative adversarial networks. In CVPR, 2018.

[14] Kaiming He, Xiangyu Zhang, Shaoqing Ren, and Jian Sun. Deep residual learning for image recognition. In CVPR, 2016.

[15] Joey Hong, Benjamin Sapp, and James Philbin. Rules of the road: Predicting driving behavior with a convolutional model of semantic interactions. In CVPR, 2019.

[16] Yedid Hoshen. VAIN: Attentional multi-agent predictive modeling. arXiv preprint arXiv:1706.06122, 2017.

[17] Diederik P Kingma and Jimmy Ba. Adam: A method for stochastic optimization. arXiv preprint arXiv:1412.6980, 2014.

[18] Thomas Kipf, Ethan Fetaya, Kuan-Chieh Wang, Max Welling, and Richard Zemel. Neural relational inference for interacting systems. In ICML, 2018.

[19] Robert Krajewski, Julian Bock, Laurent Kloeker, and Lutz Eckstein. The highd dataset: A drone dataset of naturalistic vehicle trajectories on german highways for validation of highly automated driving systems. In ITSC, 2018.

[20] Charles R Qi, Hao Su, Kaichun Mo, and Leonidas J Guibas. Pointnet: Deep learning on point sets for $3 \mathrm{~d}$ classification and segmentation. In CVPR, 2017.
[21] Charles Ruizhongtai Qi, Li Yi, Hao Su, and Leonidas J Guibas. Pointnet++: Deep hierarchical feature learning on point sets in a metric space. In NIPS, 2017.

[22] Alec Radford, Jeff Wu, Rewon Child, David Luan, Dario Amodei, and Ilya Sutskever. Language models are unsupervised multitask learners. 2019.

[23] Nicholas Rhinehart, Rowan McAllister, Kris Kitani, and Sergey Levine. PRECOG: Prediction conditioned on goals in visual multiagent settings. In ICCV, 2019.

[24] Alexandre Robicquet, Amir Sadeghian, Alexandre Alahi, and Silvio Savarese. Learning social etiquette: Human trajectory understanding in crowded scenes. In ECCV, 2016.

[25] Weijie Su, Xizhou Zhu, Yue Cao, Bin Li, Lewei Lu, Furu Wei, and Jifeng Dai. V1-bert: Pre-training of generic visual-linguistic representations. arXiv preprint arXiv:1908.08530, 2019.

[26] Chen Sun, Per Karlsson, Jiajun Wu, Joshua B Tenenbaum, and Kevin Murphy. Stochastic prediction of multi-agent interactions from partial observations. In ICLR, 2019.

[27] Chen Sun, Austin Myers, Carl Vondrick, Kevin Murphy, and Cordelia Schmid. VideoBERT: A joint model for video and language representation learning. In ICCV, 2019.

[28] Chen Sun, Abhinav Shrivastava, Carl Vondrick, Rahul Sukthankar, Kevin Murphy, and Cordelia Schmid. Relational action forecasting. In $C V P R, 2019$.

[29] Charlie Tang and Russ R Salakhutdinov. Multiple futures prediction. In NeurIPS. 2019.

[30] Ashish Vaswani, Noam Shazeer, Niki Parmar, Jakob Uszkoreit, Llion Jones, Aidan N Gomez, Łukasz Kaiser, and Illia Polosukhin. Attention is all you need. In NIPS, 2017.

[31] Petar Veličković, Guillem Cucurull, Arantxa Casanova, Adriana Romero, Pietro Liò, and Yoshua Bengio. Graph attention networks. In ICLR, 2018.

[32] Raymond A. Yeh, Alexander G. Schwing, Jonathan Huang, and Kevin Murphy. Diverse generation for multi-agent sports games. In $C V P R, 2019$.

[33] Eric Zhan, Stephan Zheng, Yisong Yue, Long Sha, and Patrick Lucey. Generative multi-agent behavioral cloning. arXiv:1803.07612, 2018. 